\title{
Drugs, chemicals and nutrition during pregnancy: impact on fetal, neonatal and adult health
}

Professor David Barker's hypothesis ${ }^{1}$ that maternal nutrition during pregnancy influences health in adult life suggested that exposure to drugs, chemicals and nutrition during pregnancy can have more subtle effects on fetal, neonatal and adult health than some of the major birth defects linked to drug exposures. ${ }^{2}$ It is known that drugs administered to the mother or chemicals in the environment can, to varying degrees, cross the placenta depending on their structure, lipid solubility, polarity and molecular weight. ${ }^{3-5}$ Therefore, exposures to particular drugs at critical periods of fetal development are known to result in defects; ${ }^{2,6}$ however, less is known about how exposure to drugs (over the counter, prescription and illicit), hormones and chemicals ${ }^{7}$ alter fetal development.

This issue focusses on maternal use of, and the effects of a range of substances including selective serotonin reuptake inhibitors (SSRIs), alcohol, cannabis, opioid maintenance pharmacotherapies, tobacco, testosterone, isoflavone and bisphenol A (BPA) on fetal and postnatal health. These studies have been performed in humans and a range of animal models in order to investigate lung function, heart development, ovarian reserve, bone mass, the risk of obesity and potential impact on cognitive development.

\section{Drugs of abuse during pregnancy}

Because of associated stigma and perceived consequences of losing a child into the welfare system, women are unlikely to voluntarily reveal substance use issues during pregnancy., Despite this, screening for substance use is often overlooked during the perinatal period. Health practitioners who manage women during this period often feel unequipped to screen for substance use because of a lack of education provided to them. Furthermore, if issues do arise during the screening process, health practitioners can feel under-resourced about where to direct women if substance use issues are exposed..$^{10}$ However, the consequences of substance use and abuse during pregnancy, as reported in the articles by Kenna et al. and Gordon et al., far outweigh the challenges presented by screening.

The article presented by Hotham et al. highlights the limitations of biomarker screening for substance use and the benefits of self-report screening tools. Hotham summarizes that self-report screening tools that assume maternal substance use behavior, and query pre-pregnancy use, are likely to result in the most honest responses. Alcohol and tobacco use are also highly indicative of illicit substance use and therefore screening for these two substances can be viewed as a lead in, to elicit a positive response for illicit substances use. These key aspects may assist health practitioners to be better informed and equipped and to ensure screening for substance use during pregnancy becomes part of standard obstetric care.

Despite the recent campaigning in Australia for 'no alcohol being the safest option during pregnancy', ${ }^{11}$ Fetal Alcohol Spectrum Disorder and Fetal Alcohol Syndrome as a result of low and high alcohol exposure during pregnancy, respectively, is still not uncommon. Through use of comprehensive sheep models, Kenna et al. present a review of the detrimental effects of late gestation alcohol exposure on the developing fetus, and the potential risk of disease and organ dysfunction that may occur later in life as a result.

If substance use does continue throughout pregnancy, as well as the potential detrimental developmental effects on the fetus, the exposed infant can also develop Neonatal Abstinence Syndrome (NAS). NAS results from the cut-off of supply of the drug that the infant was exposed to in pregnancy and if not adequately managed, in extreme cases can lead to infant mortality. ${ }^{12,13}$ Gordon et al. present one of the first studies to report the ineffectiveness of one of the most aggressive morphine treatment regimens that is used worldwide to manage NAS in infants exposed to opioid maintenance pharmacotherapies during pregnancy. Infants exposed to opioid maintenance pharmacotherapies have reduced neural development ${ }^{14}$ and it is unclear as to whether or not this is as a result of direct exposure to the drug, or mismanaged NAS.

\section{Exposure to chemicals during pregnancy}

It is not only illicit substances that can have detrimental effects on the developing fetus, the newborn and the adult later in life. Environmental toxins including pollution and chemicals used in everyday products are known to cause birth defects; however, their role in altering the normal trajectory of fetal development is less clear. ${ }^{15}$ Exposure to everyday chemicals that leach into the environment, such as BPA, from products such as food and water containers, have the potential to cause adverse outcomes in adults when exposure occurs during the perinatal period. ${ }^{7}$ Cagampang et al. examined cardiometabolic outcomes in adult mice following daily administration of BPA during the perinatal period from gestational days 11 to 19 . It was observed that prenatal exposure to BPA increased the potential of cardiovascular and metabolic dysfunction later in life as a result of altered growth trajectory, smaller kidneys, increased adiposity, impaired glucose homeostasis and raised systolic blood pressure in both female and male offspring. 


\section{Depression and use of antidepressants during pregnancy}

Approximately $96 \%$ of pregnant women use prescription and over-the-counter drugs in Australia. ${ }^{16}$ Exposure of the fetus to these drugs depends on dose, duration of treatment, the ability of the drug to cross the placenta and the ability for the drug to be removed from the fetal circulation. P-glycoprotein is a drug transporter that assists in the removal of drugs, including SSRIs, from the fetal circulation but its expression may be altered by fetal growth, ${ }^{17}$ which may be of concern because there is controversy about whether or not maternal SSRI exposure reduces fetal growth and thus birth weight. ${ }^{18-20}$ Studies suggest that while $\sim 7-13 \%$ of pregnant women experience depression, ${ }^{21}$ only $\sim 3 \%$ of pregnant women are treated with antidepressants during pregnancy. ${ }^{22}$ This is significant, as depression during pregnancy impacts on women and their families and the health of their unborn child (HALL). Specifically, depression itself has been shown to increase the risk of preterm delivery and low birth weight. ${ }^{23}$ In analyzing the effects of SSRI exposure during pregnancy on the incidence of being overweight in childhood, Grzeskowiak et al. has included a group of women who report psychiatric illness but are not treated with antidepressants. This experimental group provides novel insight into the effects of depression during pregnancy on childhood weight separately from the effects of SSRIs during pregnancy on childhood weight. Interestingly, this study suggests that there is a sex effect of maternal SSRI treatment on the risk of obesity in childhood, with female offspring exposed to SSRIs less likely to be obese than female offspring of nonexposed mothers with or without a psychiatric illness. Hall goes on to discuss the impact of postnatal depression on new mothers, their babies and their families. These issues are being addressed in Australia by the implementation of the National Perinatal Depression initiative.

\section{Maternal and neonatal nutrition}

In addition to maternal nutrition before $e^{24,25}$ and during pregnancy, ${ }^{26,27}$ nutrition during the neonatal period through breastfeeding or formula feeding is equally important and controversial. In cases where formula is used, much attention has focussed on optimizing the micro- and macro-nutrient content. Soy protein, a rich source of isoflavones, is a main component of many formulas. Dinsdale et al. show that supplementation with isoflavones in the neonatal period improves bone density some 4 months later. Furthermore, exposure to high testosterone prenatally results in accelerated ovarian follicle depletion at 5 months of age but more follicles at 21 months of age in sheep. Interestingly, these changes were not accentuated by obesity and thus the loss of cyclicity in obese women may not be due to loss of ovarian reserve.

\section{Summary}

The importance of screening for illicit drug use and tracking of both prescription and drug consumption patterns during pregnancy is important in mitigating the known adverse effects of specific drug use and exposure during pregnancy on fetal, neonatal and long-term adult health. This screening should be extended to understanding the implications of nutrition, amount and both micro- and macro-nutrient content, and exposure to environmental toxins on fetal, neonatal and adult health. Advances in data linkage provide opportunities to track exposures and outcomes in a way that was not possible even a decade ago. Implementation of national initiatives to mitigate the effects of disease during pregnancy is also required.

\section{J. L. Morrison}

Early Origins of Adult Health Research Group, School of Pharmacy and Medical Sciences, University of South Australia, Adelaide, South Australia

A. Gordon

School of Nursing and Midwifery, Sansom Institute for Health Research, University of South Australia, Adelaide, South Australia

\section{References}

1. Barker DJ, Winter PD, Osmond C, Margetts B, Simmonds SJ. Weight in infancy and death from ischaemic heart disease. Lancet. 1989; 2, 577-580.

2. Mitchell AA. Adverse drug reactions in utero: perspectives on teratogens and strategies for the future. Clin Pharmacol Therapeut. 2011; 89, 781-783.

3. Audus KL. Controlling drug delivery across the placenta. Eur J Pharmaceut Sci. 1999; 8, 161-165.

4. Ceckova-Novotna M, Pavek P, Staud F. P-glycoprotein in the placenta: expression, localization, regulation and function. Reprod Toxicol. 2006; 22, 400-410.

5. Gordon AL, Lopatko OV, Somogyi AA, Foster DJ, White JM. (R)- and (S)-methadone and buprenorphine concentration ratios in maternal and umbilical cord plasma following chronic maintenance dosing in pregnancy. Br J Clin Pharmacol. 2010; 70, 895-902.

6. Ward RM. Difficulties in the study of adverse fetal and neonatal effects of drug therapy during pregnancy. Semin Perinatol. 2001; 25, 191-195.

7. Grandjean P, Bellinger D, Bergman A, et al. The faroes statement: human health effects of developmental exposure to chemicals in our environment. Basic Clin Pharmacol Toxicol. 2008; 102, 73-75.

8. Bessant J. Habit and habitat: housing, bovernment policy, drugs, and pregnant women. Aust J Primary Health. 2004; 10, 9-20.

9. Roberts SCM, Nuru-Jeter A. Women's perspectives on screening for alcohol and drug use in prenatal care. Women's Health Issues. 2010; 20, 193-200.

10. Tandon SD, Parillo KM, Jenkins C, Duggan AK. Formative evaluation of home visitors' role in addressing poor mental health, domestic violence, and substance abuse among lowincome pregnant and parenting women. Matern Child Health J. 2005; 9, 273-283. 
11. National Health and Medical Research Council. Australian Guidelines to Reduce Health Risks from Drinking Alcohol, 2009. Commonwealth of Australia: Canberra, pp. 1-181.

12. Jones HE, Finnegan LP, Kaltenbach K. Methadone and buprenorphine for the management of opioid dependence in pregnancy. Drugs. 2012; 72, 747-757.

13. Kaltenbach K, Berghella V, Finnegan L. Opioid dependence during pregnancy. Effects and management. Obstet Gynecol Clin North Am. 1998; 25, 139-151.

14. Whitham JN, Spurrier NJ, Sawyer MG, et al. The effects of prenatal exposure to buprenorphine or methadone on infant visual evoked potentials. Neurotoxicol Teratol. 2010; 32, 280-288.

15. Schug TT, Janesick A, Blumberg B, Heindel JJ. Endocrine disrupting chemicals and disease susceptibility. J Steroid Biochem Mol Biol. 2011; 127, 204-215.

16. Henry A, Crowther C. Patterns of medication use during and prior to pregnancy: the MAP study. Aust N Z J Obstet Gynaecol. 2000; 40, 165-172.

17. Soo PS, Hiscock J, Botting KJ, et al. Maternal undernutrition reduces P-glycoprotein in guinea pig placenta and developing brain in late gestation. Reprod Toxicol. 2012; 33(3), 374-81.

18. Grzeskowiak LE, Gilbert AL, Morrison JL. Investigating outcomes following the use of selective serotonin reuptake inhibitors for treating depression in pregnancy: a focus on methodological issues. Drug Saf. 2011; 34, 1027-1048.

19. Colvin L, Slack-Smith L, Stanley FJ, Bower C. Dispensing patterns and pregnancy outcomes for women dispensed selective serotonin reuptake inhibitors in pregnancy. Birth defects research. Part A, Clin Mol Teratol. 2011; 91, 142-152.
20. Lund N, Pedersen LH, Henriksen TB. Selective serotonin reuptake inhibitor exposure in utero and pregnancy outcomes. Arch Pediatr Adolesc Med. 2009; 163, 949-954.

21. Bennett HA, Einarson A, Taddio A, Koren G, Einarson TR. Prevalence of depression during pregnancy: systematic review. Obstet Gynecol. 2004; 103, 698-709.

22. Grzeskowiake LE, Gilbert AL, Morrison JL. Hospital pharmacy dispensing records for pharmacoepidemiology research into late gestation exposure to antidepressants. J Pharm Practice Res. 2010; 40, 265-268.

23. Steer RA, Scholl TO, Hediger ML, Fischer RL. Self-reported depression and negative pregnancy outcomes. J Clin Epidemiol. 1992; 45, 1093-1099.

24. Zhang S, Rattanatray L, MacLaughlin SM, et al. Periconceptional undernutrition in normal and overweight ewes leads to increased adrenal growth and epigenetic changes in adrenal IGF2/H19 gene in offspring. FASEB J. 2010; 24, 2772-2782.

25. Zhang S, Rattanatray L, Morrison JL, et al. Maternal obesity and the early origins of childhood obesity: weighing up the benefits and costs of maternal weight loss in the periconceptional period for the offspring. Exp Diabetes Res. 2011; 2011, 585749.

26. McMillen IC, Robinson JS. Developmental origins of the metabolic syndrome: prediction, plasticity, and programming. Physiol Rev. 2005; 85, 571-633.

27. Edwards LJ, McMillen IC. Maternal undernutrition increases arterial blood pressure in the sheep fetus during late gestation. J Physiol. 2001; 533(Pt 2), 561-570. 